\title{
Licensed Shared Access for 5G: which auction mechanism to choose?
}

\author{
Ayman Chouayakh*, Aurélien Bechler*, Isabel Amigo ${ }^{\dagger}$, Loutfi Nuaymi ${ }^{\dagger}$, Patrick Maillé ${ }^{\dagger}$ \\ *Orange Labs, Chatillon, France \\ $\dagger$ IMT Atlantique, Brest, Nantes and Rennes, France \\ Email:\{ayman.chouayakh, aurelien.bechler\}@orange.com \\ Email: \{isabel.amigo, loutfi.nuaymi, patrick.maille\}@imt-atlantique.fr
}

\begin{abstract}
Licensed Shared Access (LSA) is a complementary solution allowing Mobile Network Operators (MNOs) to use another incumbent's frequency spectrum after obtaining a proper license from the regulator.

Using auctions to allocate those LSA-type licenses is a natural approach toward an efficient use of spectrum, by controlling the incentives for MNOs to declare their true valuation for the spectrum and allocating it to those who value it the most. A specificity of LSA licenses lies in the interactions among buyers, due to possibly overlapping coverage areas, this allows for allocating the same spectrum to several MNOs.

In this paper, we review the existing mechanisms taking into account such radio interference constraints, propose new ones, and compare their performance. We show how to increase the revenue, while maintaining truthful-telling, of all-or-nothing auction mechanisms by introducing a reserve price per bidder. We also investigate extensions of those mechanisms, namely when the management of interference among base stations is more subtle than partitioning base stations into groups of non-interfering base stations. For each mechanism, we show how to optimize a trade-off between expected fairness, expected revenue and expected efficiency by carefully working with groups and reserve prices. Simulations suggests that the extension of those mechanisms may lead to increase an indicator combining allocation fairness, social welfare and seller's revenue by more than $20 \%$.
\end{abstract}

\section{INTRODUCTION}

Accommodating exploding mobile data traffic is among the greatest challenges for fifth generation (5G) networks [1]. Dealing with that traffic indeed requires an optimal utilization of spectrum, but currently some holders of a licensed spectrum (e.g., militaries, satellites, some commercial users) do not always use all their frequencies-usage varies with time and geographical location-, hence there is some room for improvement, which has given rise to the proposal of the concept of dynamic spectrum access (DSA) [2].

DSA refers to the situation in where a primary user, who has an exclusive right to use the band, shares his bandwidth with a secondary user. Secondary users must allow the primary user to use his spectrum without disrupting it. For this, these systems typically use cognitive radio [3]: secondary users-Mobile Networks Operators (MNOs) in our context-can intelligently detect those communication channels that are in use and those that are not, and move to unused channels. However, for MNOs this approach is risky because neither the access to spectrum nor the quality of service (protection from interference) are guaranteed.

In November 2011, in order to support the deployments of 5G systems [4], the Radio Spectrum Policy group (RSPG) has proposed a new sharing concept called Licensed Shared Access (LSA) [5]. That concept involves three stakeholders: the incumbent user, the secondary user which is called LSA licensee, and the regulator [2]. Contrary to DSA, under the LSA approach, the secondary user needs to obtain a license from the regulator before accessing the spectrum of the incumbent. The license includes the conditions of sharing, in particular in terms of time, frequency and geographic region. The LSA concept guarantees to the incumbent and to the LSA licensee a certain level of QoS specified in the LSA license. The LSA licensee is typically an MNO, we shall thus use the terms MNO, (network) operator, or LSA licensee interchangeably. Likewise, we shall use the terms regulator, seller and auctioneer interchangeably.

Deploying an LSA system requires the introduction of two new architectural building blocks [6], as shown in Fig. 1: the LSA repository and the LSA controller. The

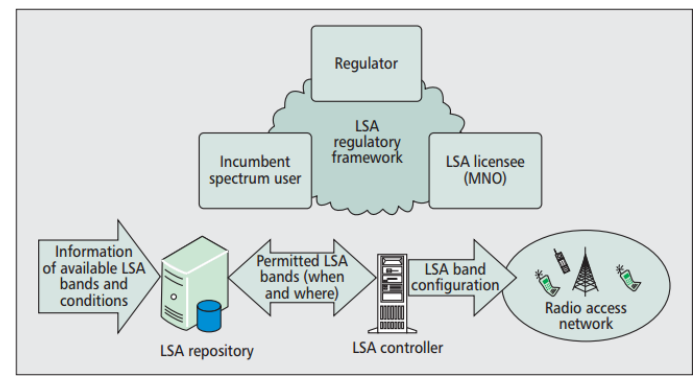

Fig. 1: Overview of the LSA concept (taken from [2])

LSA repository is a database which contains information about LSA spectrum bands together with their conditions of sharing. It is controlled by the regulator and the incumbent, and is required to deliver the information on spectrum availability based on the incumbent spectrum use and associated conditions for sharing. The LSA controller resides in the network operator's domain and controls the access 
to the incumbent's spectrum by following the instructions received from the LSA repository. Each MNO has to have his own LSA controller. Several trials of the LSA concept have taken place in Europe ${ }^{1}$ and have shown its applicability. LSA is now under the final stages of standardization and field validation [7] as regards the technical aspects, but the specifics of how to allocate and price spectrum among several potential secondary users remain open.

The LSA concept involves two major differences with regard to the allocation of $3 \mathrm{G}$ or $4 \mathrm{G}$ spectrum to operators (which already uses auction schemes). First, the allocation needs to work at a faster time scale, since the availability of LSA spectrum will be changed by the incumbent via the LSA repository, possibly several times per hour, and the regulator has to allocate the LSA spectrum for potential LSA licensees as soon as the incumbent releases his spectrum in order to improve the use of the spectrum. Second, spatial re-usability (MNOs who do not interfere can use the same spectrum bands simultaneously), should be leveraged. We will in particular consider a scenario in which multiple base stations of different operators compete for LSA spectrum at a defined period of time in a particular geographical area; no two interfering base station should be allocated the same spectrum, which is ensured by dividing MNOs into groups as will be detailed in Section 3 .

A key objective for LSA is to allocate the spectrum in the most efficient way, so as to maximize the resulting value to the market. Since the LSA ecosystem involves several actors (incumbent and MNOs) with nonaligned objectives, one needs to define allocation and pricing schemes that are robust to manipulation; hence the focus on auctions for that task. To the best of our knowledge, there are only a few research studies on auction mechanism design focused on the LSA context. This paper aims at analyzing and comparing auction schemes introduced in the literature for the specific LSA context under different scenarios, as well as benefiting from more general results on auctions ([8], [9]) to propose alternative mechanisms. To compare mechanisms, we apply the commonly used efficiency and fairness measures ([9], [10]), in addition to the fulfillment of properties such as incentive compatibility (truthfulness) which intuitively means that reporting true valuation as a bid maximizes one's payoff, and individual rationality which ensures participants a non-negative payoff.

The rest of this paper is organized as follows: after summarizing the paper's contributions, in Section 2 we define what an auction mechanism is and describe some of its desirable properties, while the system model we consider is introduced in Section 3. Section 4 contains the main contributions of this paper: under the assumptions made in the literature, we review some proposed mechanisms, adapting one to ensure truthfulness. We also adapt them to include a per-buyer reserve price set by the auctioneer while maintaining incentive properties, and numerically compare those mechanisms with others from the literature in terms of efficiency, revenue, and fairness. Section 5 investigates the relaxation of a key assumption in the model: while the mechanisms partition the base stations into separate groups and allocate spectrum among groups, we consider

1. https://www.cept.org/ecc/topics/lsa-implementation allowing overlapping groups (groups still covering all base stations, but not necessarily in a partition). This relaxation may improve efficiency of the allocation but complicates the mechanism analysis (ensuring truthfulness becomes harder). Indeed, the payment of each base station which belongs to the winner group is a function of bids of other losing group(s). When relaxing that assumption, a winner base station could be also in another losing group(s) therefore its bid may impact its final payment. Finally, we provide some concluding remarks and suggest some perspective for future work in Section 6.

\section{Contributions of the paper}

The first auction mechanism which was proposed in the LSA context is named LSAA [11]; we have shown that this mechanism is not incentive compatible, and proposed PAM [12], a truthful auction mechanism that outperforms LSAA in terms of revenue and fairness. However, PAM relies on the assumption that players will accept even an infinitesimal portion of the LSA bandwidth. All the mechanisms proposed in the literature specifically for LSA -reviewed in Section 3.5- except PAM rely on the assumption that each base station must belong to one and only one group (for otherwise they are not incentive-compatible). The main contributions of this paper can be summarized as follows:

- We show how to adapt PAM to a more realistic setting: the regulator sets a minimum fraction $\alpha$ : each player must get at least that amount or he gets nothing.

- We design two new truthful spectrum auction mechanisms from the non-truthful LSAA, named TLSAA and TLSAA2. We prove that the revenue generated by TLSAA2 equals the second-highest bid and is thus may be an attractive choice from the auctioneer's viewpoint.

- We show how to increase the revenue provided by the proposed mechanisms, while maintaining truthtelling, by introducing a reserve price per bidder.

- We give the regulator more flexibility in the group construction by allowing each base-station to be in multiple groups, and by showing how to adapt the payment rules of the previous mechanisms, when possible, for any group configuration, to maintain truthfulness bidding without modifying the allocation rule.

\section{AUCTION MECHANISMS AND DESIRABLE PROP- ERTIES}

In this section, we provide the definition of an auction mechanism, and of possible properties (goals) that a regulator may want the mechanism to satisfy. Note that each designer of an auction mechanism may be interested in a particular subset of properties.

\subsection{Auction mechanisms}

We consider $N$ strategic agents ("players", or "bidders") wishing to acquire some-possibly divisible-goods. 
An auction mechanism takes some bids $b=\left(b_{1}, . ., b_{N}\right)$ submitted by the players under a predetermined format, and based on those bids, returns:

- an allocation of the good(s) among the bidders,

- a payment vector $p=\left(p_{1}, . ., p_{N}\right)$, where $p_{i}$ is the (possibly negative) price that player $i$ is charged.

In this paper, we limit ourselves to direct auction mechanisms, i.e., mechanisms where the bid format contains all the information to build the bidder's utility function. This is actually without loss of generality, due to the Revelation Principle [8], and for our model will translate into bidders declaring the price they are willing to pay per unit of spectrum.

The objective of each player $i$ is to maximize his own objective function, which we call his utility and denote by $u_{i}$ [13]. Since that utility depends on allocations and prices (computed based on bids), it is reasonable to assume that players will try to bid strategically to maximize their utility, hence the need for the mechanism to take that behavior into account.

\subsection{Desirable properties for an auction scheme}

In this paper, we will consider the following properties that a mechanism may satisfy, which are the most used in the literature [14], [15].

As for any multi-constraint problem, it is not possible to jointly satisfy all properties, hence the auction designer has to set a trade-off between them.

\subsubsection{Revenue maximization}

The revenue of the regulator, Rev, is the sum of payments of all players:

$$
\operatorname{Rev}=\sum_{i=1}^{N} p_{i} .
$$

A mechanism maximizing that metric is desirable from the seller's point of view; such mechanisms are studied in particular in [16].

\subsubsection{Truthfulness}

A mechanism is truthful or incentive compatible if and only if for each player $i$, declaring truthfully one's preferences maximizes one's utility given any fixed bids of the other players.

\subsubsection{Individual rationality}

This property means that a player has a bidding strategy that ensures him to get a non-negative utility, hence he is always better off participating in the auction than staying out of the mechanism.

\subsubsection{Fairness of the allocation}

There exist several measures of fairness [17]. In this paper, we will use Jain's index which is given by:

$$
J(\alpha)=\frac{\left(\sum_{i=1}^{N} \alpha_{i}\right)^{2}}{N \sum_{i=1}^{N} \alpha_{i}^{2}},
$$

with $\alpha_{i}$ the quantity of good allocated to player $i$. This index is a continuous function of the allocations, with values in $\left[\frac{1}{N}, 1\right]$ : it achieves its maximum 1 if all players obtain the same amount, and is minimum and equal to $\frac{1}{N}$ if one and only one player obtains some good. As another reference, a situation in which $a \%$ of users receive equal allocation and the remaining $(100-a) \%$ receive zero [18] gives a Jain index of $a / 100$. Motivated by those features we will use this index to measure the fairness of a mechanism's allocation.

\subsubsection{Efficiency}

We define efficiency as as the sum of the valuations served $\sum_{i=1}^{N} \alpha_{i} v_{i}$ [19], where $v_{i}$ is the valuation of player $i$ for the good. This means that the social value of the good being sold equals the maximum of the potential buyers' individual valuations [20].

\subsection{Truthfulness and minimal price}

Truthfulness is very important because it reduces the complexity of the game for players, since the strategies to play are very simple (just declare one's preferences). In particular, that property induces some fairness in participation, in the sense that wealthier players cannot get an edge over competitors by implementing costly measures to optimize their bidding strategy. Also, this property is desirable from the auctioneer point of view: if one objective is efficiency, it is simpler to base the allocation optimization on real utilities rather than unfaithful ones.

Luckily, when bidders' allocation is one-dimensional, this property can be guaranteed in a quite general setting: Myerson indeed showed in a lemma [16] that an allocation rule $\alpha_{i}\left(b_{1}, \ldots, b_{N}\right)$ is implementable (there is a truthful payment rule that can be associated to it) if and only if it is monotone. An allocation rule is monotone if for each player $i$ and bids $b_{-i}, \alpha_{i}\left(b_{i}, b_{-i}\right)$ is non-decreasing in $b_{i}$, where $b_{-i}=\left(b_{1}, . ., b_{i-1}, b_{i+1}, . ., b_{N}\right)$. In addition, if we add the constraint that a zero bid implies a zero payment, the payment rule is unique.

Roughgarden details that payment rule in a case that is particularly relevant for us [21]: given a piecewise constant monotone allocation curve as shown in Fig. 2a, each player $i$ should pay a price as a function of the corresponding breaking points (points at which $i$ 's allocation changes) in the range $\left[0, b_{i}\right]$. Specifically, if there are $X$ breaking points $\left(z_{j}\right)$ then the payment is given by:

$$
p_{i}\left(b_{i}, b_{-i}\right)=\sum_{j=1}^{X} z_{j} \cdot\left(\text { jump in } \alpha\left(., b_{-i}\right) \text { at } z_{j}\right) .
$$

This price corresponds to the greyed surface in Fig. 2a ( $X=$ $3)$. In particular, if there is one and only one indivisible item, i.e., the allocation is either 1 or 0 as shown in Fig. $2 b$ then there is one and only one breaking point for each player, that is his minimum bid to win the auction. As an example, in the second-price auction the breaking point for each player is the maximum bid of the other players.

In addition, without loosing truthfulness and in order to protect himself from low revenues, the auctioneer may introduce a "reserve price per bidder", imposing in the allocation rule that bids strictly below that price be allocated 


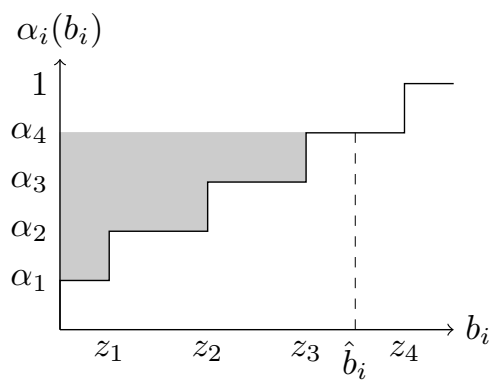

(a) Divisible item

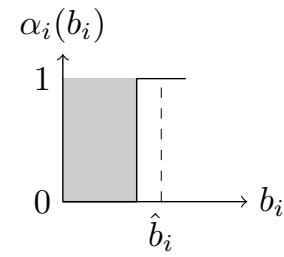

(b) Indivisible item
Fig. 2: Some piecewise constant monotone allocation curves (solid curves). The grey area represents the price paid when the bid $b_{i}$ has value $\hat{b}_{i}$.

no resource [22]. By applying Myerson's result above (since the allocation is still monotone), this involves that the perunit revenue from each player is at least that reserve price: any bid strictly below it leading to a null allocation, the breaking point(s) for each winning player must at least equal that reserve price.

\section{System MODEL FOR LSA AUCTIONS}

In this section, we instantiate the general auction framework to the specific context of LSA auctions. More specifically, we describe our model for players (here, operators and the regulator) preferences, and explain how the interference among coverage areas is managed, through the definition of groups of base stations. We then describe the general working of an LSA auction scheme and present some auction mechanisms which were proposed in that context.

\subsection{Preferences of operators}

We consider $N$ base stations in competition to obtain spectrum under the LSA scheme. A given quantity of available spectrum is auctioned, which we normalize to 1 w.l.o.g., and which we assume can be arbitrarily split among base stations. We assume that each base station $i=1, \ldots, N$ has a quasi-linear utility function, with a constant marginal valuation $v_{i}$ for spectrum: if it obtains a fraction $\alpha_{i}>0$ of the available bandwidth and pays $p_{i}$, his utility is:

$$
u_{i}\left(b_{i}, b_{-i}, v_{i}\right)=\alpha_{i}\left(b_{i}, b_{-i}\right) v_{i}-p_{i}\left(b_{i}, b_{-i}\right)
$$

Otherwise his utility is zero. Notice that we have assumed indistinguishable channel properties [23], [24], i.e., base stations are only sensitive to the amount of bandwidth-and not to the specific bands-they can use.

Under those assumptions, the preferences of base station $i$ are completely characterized by the value $v_{i}$, hence if each base station is controlled by a different player (operator), a direct auction mechanism would simply ask each one to declare one's $v_{i}$. The truthfulness property would then translate into players not being able to do better than proposing a bid $b_{i}=v_{i}$, i.e.,

$$
u_{i}\left(v_{i}, b_{-i}, v_{i}\right) \geq u_{i}\left(b_{i}, b_{-i}, v_{i}\right) \forall b_{-i} . b_{i},
$$

\subsection{The utility of the regulator}

In this paper, we assume the regulator is sensitive to the revenue from the auction, the allocation's fairness and efficiency. More specifically, we suppose that, given a mechanism, the normalized utility of the regulator $U_{\text {Reg }}$ is of the form

$$
U_{\text {Reg }}=\beta_{1} \frac{\operatorname{Rev}}{\operatorname{Rev}^{\max }}+\beta_{2} J(\alpha)+\beta_{3} \frac{\mathrm{Eff}}{\mathrm{Eff}^{\max }},
$$

where $\beta_{1}$ is the the weight that the regulator puts on revenue, $\beta_{2}$ is the weight that he puts on fairness and $\beta_{3}$ is the weight that he puts on efficiency. $\boldsymbol{\beta}=\left(\beta_{1}, \beta_{2}, \beta_{3}\right)$, $\beta_{1}+\beta_{2}+\beta_{3}=1, \operatorname{Rev}^{\max }$ and Eff $^{\max }$ are respectively the maximum revenue and maximum efficiency over the set of candidate mechanisms that we use to normalize the revenue criterion in (2).

\subsection{Grouping operators before the auction}

We consider a scenario in which $N$ base stations of different operators compete over the LSA spectrum. Two base stations can use the same bandwidth simultaneously if they do not interfere with each other. This can be captured in a model by using an interference graph. Fig. 3 shows an example of an interference graph: base stations are represented by vertices, an edge between two vertices means that those base stations interfere. For example, in Fig. 3 base stations $\{3,5\}$ can use the same fraction of bandwidth simultaneously. The competition between the $N$ base stations is transformed into a competition between $M$ groups in such a way that two base stations in the same group $k$ (the set of base stations in that group is denoted by $g_{k}$ ) do not interfere, hence the spectrum allocated to a group is used by all the members of the group. The group creation is performed by the auctioneer from the interference graph before the actual auction takes place. An example of group constitution for the interference graph of Fig. 3 is: $g_{1}=\{1,2,4,6\}$ and $g_{2}=\{3,5\}$. Another possible configuration is $g_{1}=\{1,2,4,6\}, g_{2}=\{1,2,5,6\}$ and $g_{3}=\{3,5,6\}$.

While the group formation has a non-negligible impact on the auction's outcome, in this paper (as in [11], [12], [25], [26] that also rely on groups) we assume that the groups are formed by the auctioneer, and advertised to bidders, before any bids are submitted. We indeed focus here on how to allocate the resource among groups, based on the submitted bids.

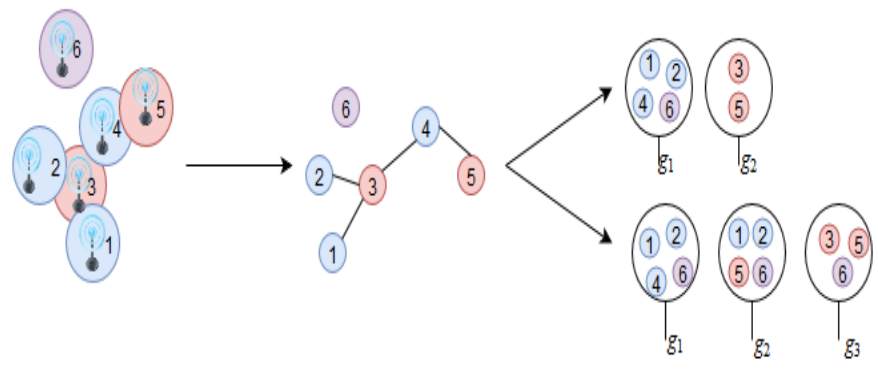

Fig. 3: Some base stations with their coverage areas (left), the corresponding interference graph (center), and two possible group configurations (right). 


\subsection{Steps of the auction}

The main steps of all the auction schemes considered in this paper are the same, and summarized as follows.

1) Group construction: from the interference graph, the regulator constructs groups under the constraint "two base stations in the same group do not interfere with each other";

2) Bid collection: bidders are asked to declare their valuation;

3) Allocation: each base station is allocated some fraction $\alpha_{i}$ of the available spectrum (specific to the mechanism used);

4) Payment: each player $i$ is charged a price $p_{i}$ (specific to the mechanism).

\subsection{State of the art}

In the following, we present some auction mechanisms that have been proposed for LSA. In each case the allocation to each group is based on its "groupbid", which is a mechanism-specific quantity. All the notations used throughout the paper are given in Table 1 . Note that for all the mechanisms in this subsection -except for PAM- the bandwidth is allocated to one and only one group: for each player $i, \alpha_{i}$ is either 1 (if $i$ is a winning player) or 0 .

\begin{tabular}{|c|l|}
\hline$R$ & minimum per bidder price set by the auctioneer \\
\hline$M$ & number of groups \\
\hline$N$ & number of base stations \\
\hline$v_{i}$ & $\begin{array}{l}\text { true valuation of base station } i \text { per bandwidth } \\
\text { unit }\end{array}$ \\
\hline$b_{i}$ & bid of base station $i$ per bandwidth unit \\
\hline$b_{-i}$ & bids of all base stations except $i$ \\
\hline$g_{k}$ & group $k$ (a set of base station indices $)$ \\
\hline$\alpha_{i}$ & fraction of spectrum allocated to base station $i$ \\
\hline$B_{k}$ & $\begin{array}{l}\text { sum of bids of } g_{k}, \\
B_{k}=\sum_{i \in g_{k}} b_{i}\end{array}$ \\
\hline$B_{\text {Tot }}$ & $\begin{array}{l}\text { sum of the total bids of all groups, } \\
B_{\text {Tot }}=\sum_{k=1}^{M} B_{k}\end{array}$ \\
\hline$B_{g}^{-i}$ & $\begin{array}{l}\text { sum of bids of groups which } i \text { belongs to except } \\
i^{\prime} \text { s bid, } B_{g}^{-i}=\left(\sum_{k=1}^{M} B_{k} \mathbb{1}_{i \in g_{k}}\right)-n_{i} b_{i}\end{array}$ \\
\hline$B_{\text {Tot }}^{-i}$ & $\begin{array}{l}\text { sum of the total bids of all groups except } i^{\prime} \text { s bid, } \\
B_{\text {Tot }}^{-i}=\left(\sum_{k=1}^{M} B_{k}\right)-n_{i} b_{i}\end{array}$ \\
\hline
\end{tabular}

TABLE 1: Notations.

\subsubsection{TAMES}

TAMES [25] computes the groupbid of each group $k$ as $\left(\left|g_{k}\right|-1\right) \min _{i \in g_{k}} b_{i}$, where $\left|g_{k}\right|$ is the cardinal of group $k$. All players of the highest-groupbid group are winners, except the one with the lowest bid of that group. Each winning player pays the same price, that is the lowest bid in their group.

\subsubsection{TRUST}

TRUST [26] works quite similarly to TAMES. It computes the groupbid as: $\left|g_{k}\right| \min _{i \in g_{k}} b_{i}$. All players of the group with the highest groupbid are winners. Winners pay equitably the second-highest groupbid (each winner pays a proportion $1 /\left|g_{k}\right|$ of it).

\subsubsection{LSAA}

In LSAA [11], bids in each group are sorted in a nonascending order. The groupbid of a group $g_{k}$ is computed as: $\max _{i \in g_{k}} \operatorname{rank}\left(b_{i}\right) b_{i}$, where $\operatorname{rank}\left(b_{i}\right)$ is the rank of player $i$ 's bid in the group. The authors define an index $j$ such that:

$$
j=\max \left\{\operatorname{rank}\left(b_{l}\right), l \in \arg \max _{i \in g_{k}}\left(\operatorname{rank}\left(b_{i}\right) b_{i}\right)\right\} .
$$

If $g_{k}$ is the winning group, then only players with rank below or equal to $j$ are winners. Winners pay the second highest groupbid equally.

For TAMES and TRUST, the allocation is based on the bidder with the lowest bid. This can extremely harm the efficiency and the revenue. For LSAA, we have shown in [12] that this mechanism is not always truthful, we therefore adapt it in two different fashions in the next subsection.

\subsubsection{VCG}

In [22], we adapted the Vickrey-Clarke-Groves (VCG) ([27][29]) mechanism to the LSA context i.e., given an efficient allocation, we derive the payment for each player of each group, eliciting truthful bidding. The principle of VCG is to allocate resources to maximize the "declared" efficiency (since computed based on submitted bids) and charge each bidder the loss of declared welfare his presence causes to the others. A way to implement VCG would be to compute the groupbid of a group $g_{k}$ as $\sum_{i \in g_{k}} b_{i}$; the winning group is then the group with the highest groupbid. If a player belongs to a losing group he pays 0 because whether he is present or not the winning group is the same. If a player belongs to the winning group $g_{\text {win }}$ with group bid $B_{\text {win }}$ then we can distinguish two cases: if his presence does not change the outcome, i.e., $B_{\text {win }}^{-i} \geq B_{\text {second }}$ (with $B_{\text {second }}$ the second-highest groupbid) then he pays 0 otherwise he pays $B_{\text {second }}-B_{\text {win }}^{-i}$. To summarize:

$$
p_{i}^{V C G}=\left[B_{\text {second }}-B_{\text {win }}^{-i}\right]^{+} .
$$

That mechanism is known to be efficient, individually rational, and truthful [9].

\subsubsection{Proportional Allocation Mechanism (PAM)}

In [12] we proposed PAM, which allocates to each group $k$ a fraction $\alpha_{k}$ of the bandwidth in proportion to the bids submitted by players belonging to that group i.e., $\alpha_{k}=\frac{\sum_{i \in g_{k}} b_{i}}{B_{\text {Tot }}}$. Each player $i$ pays an amount computed to ensure truthfulness, given by:

$$
\begin{aligned}
p_{i}^{\mathrm{PAM}}= & \frac{b_{i}+B_{g}^{-i}}{b_{i}+B_{\mathrm{Tot}}^{-i}} R+\left(B_{\mathrm{Tot}}^{-i}-B_{g}^{-i}\right) \\
& \left(\ln \left(\frac{b_{i}+B_{\mathrm{Tot}}^{-i}}{R+B_{\mathrm{Tot}}^{-i}}\right)+\frac{R+B_{\mathrm{Tot}}^{-i}}{b_{i}+B_{\mathrm{Tot}}^{-i}}-1\right),
\end{aligned}
$$

where $R$ is a reserve price per bidder set by the auctioneer, that ensures that the per-unit price paid by each bidder is at least $R$.

In [22], we have extended all the aforementioned mechanisms by introducing a reserve price $R$ per bidder while maintaining truthfulness; the general method is detailed in Proposition 4. 


\section{Proposed mechanisms}

This section introduces several alternative mechanisms that we suggest could be applied to auction LSA spectrum. We start by introducing $\mathrm{PAM}^{\alpha}$ which is an extension of PAM after introducing a minimum amount $\alpha$ i.e., if a player $i$ gets a fraction $\alpha_{i} \neq 0$ then $\alpha_{i}$ must be higher than $\alpha$. Then we present two new auction mechanism named TLSAA and TLSAA2 which are two extensions of LSAA [11]. Finally we show how to increase the revenue for any monotone and all-or-nothing allocation rule. Note that in this section, we make a key assumption, initially introduced by the schemes proposed in the literature. This assumption is the following:

Assumption A. The grouping is made such that each base station belongs to one and only one group.

In Section 5, we investigate the consequences of relaxing this assumptions.

\section{1 $P A M^{\alpha}$ : guaranteeing winners get at least a mini- mum amount of spectrum}

In the following, we show how to make the mechanism more suitable to be applied in real life, where very small portions of spectrum might not be useful for operators. The regulator fixes a minimum amount $\alpha$ : each player must get at least that amount or he gets nothing. We call this extension $\mathrm{PAM}^{\alpha}$. For the allocation, we keep excluding groups with low group-bids (we start by the one with the lowest group-bid) until obtaining a feasible allocation. Suppose there are three groups, the first group is composed of two players with bids 12 and 5 respectively. The second group is composed of one player with bid 14 and the third group is composed of one player with bid 15. Suppose that $\alpha=0.4$. We start by computing the fraction allocated to group two which is lower than $0.4\left(\frac{14}{46}<0.4\right)$. Thus, group two will be excluded and the final allocation is $\frac{17}{32}$ for group one and $\frac{15}{32}$ for group three.

In the following, we investigate $\mathrm{PAM}^{\alpha \prime}$ s efficiency.

Proposition 1. As $\alpha$ gets closer to $0.5, P A M^{\alpha}$ 's efficiency gets closer to VCG's efficiency. Moreover, when $\alpha \geq 0.5$, PAM ${ }^{\alpha}$ 's efficiency is equal to VCG's efficiency.

Proof. We denote by $M^{r}$ the number of remaining groups i.e., groups with allocation higher than $\alpha$. We can distinguish three cases:

- $\alpha<0.5$, the maximum number of remaining groups is $\left\lfloor\frac{1}{\alpha}\right\rfloor$, and that as we increase $\alpha$, we exclude groups with low group-bids. Therefore, groups with high group-bids will obtain more spectrum which leads to increase efficiency.

- $\alpha=0.5$ in this situation $M^{r}$ is either:

- 1 , when the two highest group-bids have different value. In this situation the group with the highest group-bid obtains all the available bandwidth and we obtain the same efficiency as VCG.

- 2 , when the two highest group-bids are equal. In this situation, each group obtains a fraction equal to 0.5 (each bidder pays $\frac{b_{i}}{2}$ ) and in this situation we obtain the same efficiency as VCG but we increase the fairness of the allocation.

- $\alpha>0.5$, all the available bandwidth must be allocated to one and only one group. The remaining group is the group with the highest group-bid (if there are many, we choose one randomly). Therefore we obtain the same efficiency as VCG.

We present in Appendix A the payment rule for this extension.

\subsection{TLSAA and TLSAA2 (extensions to LSAA)}

As pointed out previously, the initial design of LSAA was not truthful. We propose here two variants that are truthful, and that can also be extended by adding a reserve price, when seeking to optimize auctioneer's revenue.

\subsubsection{TLSAA}

We preserve LSAA's method of groupbid computation and allocation, but propose a new payment rule which ensures a truthful bidding: since the allocation rule is monotone, we can implement the truthful payment rule given in (1). This gives

$$
p_{i}=\min \left\{b_{i} \text { s.t. } \alpha_{i}\left(b_{i}\right)=1\right\} .
$$

We illustrate that rule with an example: suppose we have two groups with bids respectively $\{20,10,9,6,3\}$ and $\{20,8,7\}$. The first group wins the auction since it has the highest groupbid (with value 27). Let us compute the payment of the first player (the one with bid 20): by proposing a bid lower than 5.25 player 1 would be a losing player because the groupbid of his group would then be below the second groupbid 21, and by proposing a bid higher than 5.25 group 1 wins the auction. So Player 1 should pay 5.25. Note that for the second and the third player the same reasoning can be made and each one should pay 5.25, however the fourth player should pay 0 because his group is a winning group whether he is present or not (there is no breakpoint for him).

In LSAA, the revenue is given by the second highest groupbid. A question which may arise regards the revenue of this modified version of LSAA. We show below that truthfulness comes at a cost, since revenue may decrease with respect to the initial version (assuming truthful bidding).

Proposition 2. The revenue of TLSAA cannot be higher than the second-highest groupbid.

Proof. We denote by $g_{w}$ the winning group. Let us define $j^{\prime}$ such that:

$$
j^{\prime}=\max \left\{\operatorname{rank}\left(b_{i}\right), i \in g_{w} \text { and } \operatorname{rank}\left(b_{i}\right) b_{i} \geq B_{\text {second }}\right\} .
$$

Consider a player $i$ in the winning group:

- if $\operatorname{rank}\left(b_{i}\right)$ is strictly above $j^{\prime}$ then that player pays 0 , because his group always wins whatever his bid (there is no breaking point for him);

- if $\operatorname{rank}\left(b_{i}\right)$ is below $j^{\prime}$ then we can distinguish two cases: 
1) if his group remains the winning group without $i$ 's bid, that player pays 0 .

2) if his group is a loosing group if $i$ is not there (winning group only with his presence), his breaking point is exactly $\frac{B_{\text {second }}}{j^{\prime}}$.

Hence the maximum revenue is $\frac{B_{\text {second }}}{j^{\prime}} \cdot j^{\prime}=B_{\text {second }}$.

One may then wonder whether we can find an allocation rule that ensures the same revenue as TLSAA. To reach that goal, we propose TLSAA2, in which the group bid is defined as in LSAA, but we modify the allocation rule and still apply the payment rule ensuring truthful bidding, given in (1).

\subsubsection{TLSAA2}

The groupbid is calculated as in LSAA. The allocation rule is defined as follows: a winning player should not only belong to the winning group but also bid at least as high as player $j^{\prime}$ (see (7)). In turn, the payment rule is defined as follows: each winning player pays

$$
p_{i}=\frac{B_{\text {second }}}{j^{\prime}} .
$$

Through the following proposition we prove that TLSAA2 is a truthful mechanism with revenue achieved by the seller equal to $B_{\text {second }}$.

Proposition 3. TLSAA2 is truthful with revenue equal to $B_{\text {second }}$.

Proof. For the revenue, it is clear that it is equal to $\frac{B_{\text {second }}}{j^{\prime}} j^{\prime}=B_{\text {second }}$. This payment rule ensures a truthful bidding because the allocation rule is monotone (the allocation rule of TLSAA2 is just the allocation rule of TLSAA with constraint given by (7)), and the payment rule corresponds to Equation (1).

Table 2 summarizes state-of-the-art mechanisms as well as mechanisms proposed in this article for truthful bandwidth allocation auctions in the LSA context.

\subsection{How to increase revenue?}

In order to increase VCG's revenue, authors in [30] have introduced a reserve price per bidder. In a previous work in [22], we have extended that approach for other mechanisms. In the following proposition, we generalize [22] for any mechanism with a monotone and all-or-nothing allocation rule.

Proposition 4. Consider a mechanism denoted by Mec with a monotone and all-or-nothing allocation rule $\left(\alpha_{i}\right.$ is either 0 or 1 for each player i). We denote by $p^{\prime}$ the corresponding truthful payment rule. For any non-negative value $R$, the mechanism $\mathrm{Mec}^{\prime}$ defined as follows is truthful:

- the allocation rule $\alpha^{\prime}$ is simply the rule $\alpha$, ignoring all bids strictly below $R$;

- the payment rule consists in charging player $i$ a price

$$
p_{i}^{\prime}\left(b_{i}\right):= \begin{cases}\max \left\{R, p_{i}^{R}\left(b_{i}\right)\right\}, & \text { if } \alpha_{i}^{\prime}=1 \\ 0, & \text { if } \alpha_{i}^{\prime}=0,\end{cases}
$$

with $p_{i}^{R}\left(b_{i}\right)$ the price given by the original mechanism

\begin{tabular}{|c|c|c|c|}
\hline Mech. & Groupbid & Allocation & Payment \\
\hline TAMES & $\left(\left|g_{k}\right|-1\right) \min _{i \in g_{k}} b_{i}$ & $\begin{array}{l}\text { group with } \\
\text { the highest } \\
\text { groupbid } \\
\text { except the } \\
\text { one with the } \\
\text { lowest bid }\end{array}$ & $\begin{array}{c}\text { each } \\
\text { winning } \\
\text { player pays } \\
\text { the lowest } \\
\text { bid of his } \\
\text { group }\end{array}$ \\
\hline TRUST & $\left|g_{k}\right| \min _{i \in g_{k}} b_{i}$ & $\begin{array}{l}\text { group with } \\
\text { the highest } \\
\text { groupbid }\end{array}$ & $\begin{array}{c}\text { winners pay } \\
\text { the second } \\
\text { highest } \\
\text { groupbid } \\
\text { equally }\end{array}$ \\
\hline VCG & $\sum_{i \in g_{k}} b_{i}$ & $\begin{array}{l}\text { group with } \\
\text { the highest } \\
\text { groupbid }\end{array}$ & see Eq. (4) \\
\hline PAM & $\sum_{i \in g_{k}} b_{i}$ & $\begin{array}{l}\text { each group } \\
\text { obtains a } \\
\text { fraction in } \\
\text { proportion } \\
\text { to its } \\
\text { groupbid }\end{array}$ & see Eq. (5) \\
\hline PAM $^{\alpha}$ & $\sum_{i \in g_{k}} b_{i}$ & $\begin{array}{l}\text { each group } \\
\text { obtains } \\
\text { either a } \\
\text { fraction (at } \\
\text { least equal } \\
\text { to } \alpha \text { ) in } \\
\text { proportion } \\
\text { to its } \\
\text { groupbid or } \\
\text { nothing } \\
\end{array}$ & $\begin{array}{c}\text { see } \\
\text { Appendix A }\end{array}$ \\
\hline TLSAA & $\max _{i \in g_{k}} \operatorname{rank}\left(b_{i}\right) b_{i}$ & $\begin{array}{l}\text { group with } \\
\text { the highes } \\
\text { groupbid }\end{array}$ & see Eq. (6) \\
\hline TLSAA2 & $\max _{i \in g_{k}} \operatorname{rank}\left(b_{i}\right) b_{i}$ & $\begin{array}{l}\text { players (of } \\
\text { the group } \\
\text { with the } \\
\text { highest } \\
\text { groupbid) } \\
\text { and with } \\
\text { rank below } \\
j^{\prime} \text { see (7) }\end{array}$ & see Eq. (8) \\
\hline
\end{tabular}
rule where bids strictly below $R$ are ignored.
TABLE 2: Summary of truthful auction mechanisms that can be applied to allocate LSA spectrum.

Additionally, that modification ensures that the per-unit price paid by players is at least $R$.

Proof. The allocation rule $\alpha^{\prime}$ is still monotone, therefore there must exist a payment rule $p^{\prime}$ which renders the mechanism truthful.

Let us fix a player $i$ with valuation $v_{i}$. If $v_{i}<R$, bidding truthfully ensures a utility equal to 0 otherwise he obtains either a negative utility or a utility equal to 0 .

We distinguish two cases for a winning player with $v_{i}>R$ :

- $p_{i}^{R}\left(v_{i}\right) \geq R$ : this situation corresponds to the original mechanism facing only bidders with valuations above $R$, hence proposing a bid $b_{i}=v_{i}$ maximizes his utility.

- $0 \leq p_{i}^{R}\left(v_{i}\right)<R$ : bidding truthfully generates a utility $v_{i}-R$, any other bid $b_{i}$ leads to a lower utility since the bidder would either get no resource (hence utility 0 ), or still be a winner and pay at least $R$.

For a losing player, the outcome corresponds to the original mechanism Mec (now facing only bidders with valuations above $R$ ). Since Mec is truthful, and $\mathrm{Mec}^{\prime}$ only has larger payments than Mec, bidding truthfully-and losing-remains a best strategy. 
Hence, we can introduce to TLSAA and TLSAA2 a reserve price per bidder and the payment rule for each mechanism is given by (9).

\subsection{Performance evaluation}

This subsection compares the performance of the different aforementioned truthful mechanisms (i.e. TAMES, TRUST, TLSAA, TLSAA2, VCG, PAM $^{\alpha}$ ). The performance evaluation is based on simulations. We are particularly interested in average efficiency and fairness metrics, as well as in the average revenue of the auctioneer.

\subsubsection{Simulation settings}

We recall that this section considers the scenario where each base station belongs to one and only one group. We have thus fixed two groups from the interference graph of Fig. 3: $g_{1}=\{1,2,4,6\}$ and $g_{2}=\{3,5\}$. The marginal valuations of base stations are drawn from the uniform distribution over the interval $[0,100]$.

For each extended mechanism and for each reserve price $R$, we have computed the average (with respect to each metric) over 10.000 draws.

\subsubsection{Results}

Simulation results (Fig. 4) show that PAM outperforms the other schemes in terms of fairness. In terms of revenue, Fig. 5 shows that TRUST could offer the highest revenue by playing on the reserve price. In terms of efficiency, VCG is efficient by construction.

In terms of fairness, we can observe that the curve is non-increasing, this can be intuitively explained as follows: as we increase the reserve price, we increase the number of excluded players (with valuation lower than $R$ ). On the other hand, the bandwidth will be allocated for the remaining players (or for a set of the remaining players) hence we increase the gap (of allocation) between players which means Jain's index decreases.

We also observe a non-monotonicity on the average revenue and efficiency which can be explained as follows: by increasing the reserve price the revenue from a player $i$ keeps increasing until reaching $R=v_{i}$. Intuitively for efficiency, as we increase the reserve price, bidders with low valuations will be excluded and then players with higher valuations will be allocated more resource, then we increase the efficiency; however if we set a too high reserve price then many players will be excluded, reducing efficiency.

In addition for $\mathrm{PAM}^{\alpha}$, it offers higher efficiency and lower fairness compared to PAM, this is natural since some groups will be excluded (which leads to decrease fairness) and the original fraction allocated to those groups (by PAM) will be used by other groups with higher valuations (leads to increase efficiency).

Simulations results show that, as aforementioned, there is no one single mechanism which outperforms the others at all metrics. Which mechanism to chose should be thus based on the different weights given by the auctioneer to each criteria. We further investigate on it and report several simulation results on Subsection 5.2.

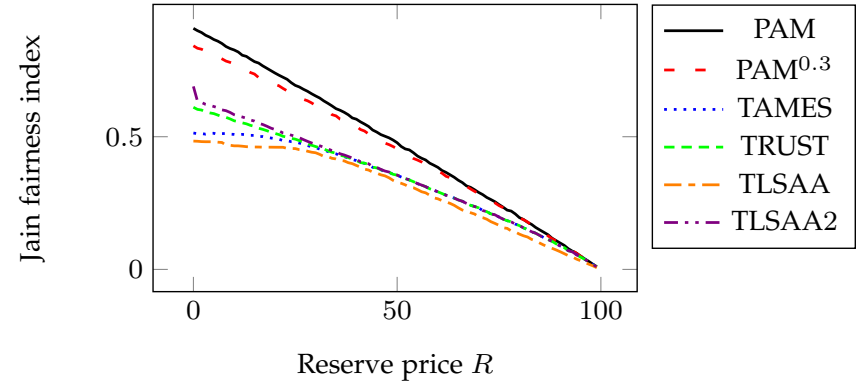

Fig. 4: Average fairness as a function of the reserve price $R$

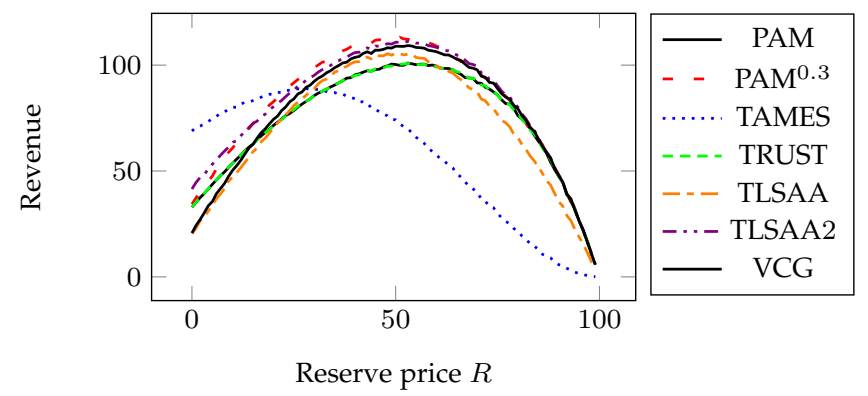

Fig. 5: Average Revenue as a function of the reserve price $R$

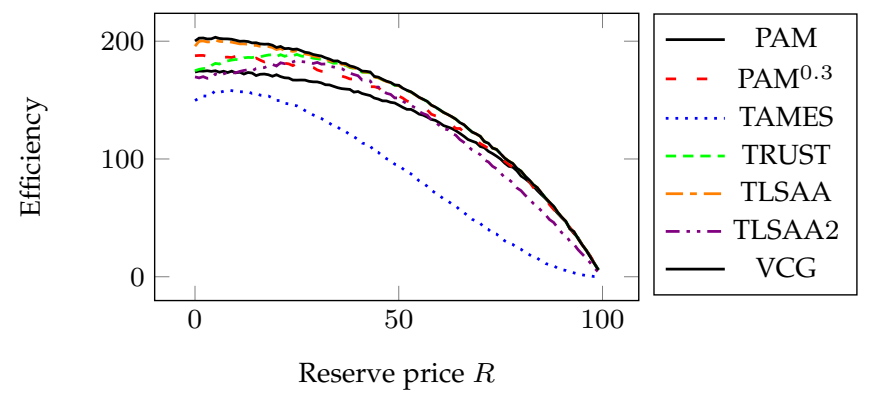

Fig. 6: Average efficiency as a function of the reserve price $R$

\section{EXTENSIONS OF THE PREVIOUS MECHANISMS: A BASE STATION CAN BELONG TO MORE THAN ONE GROUP}

In this section, we consider relaxing the assumption made previously by treating the case where the base station (BS) grouping allows a base station to belong to several groups, which should improve the efficiency of the allocation but complicates the mechanism analysis (ensuring truthfulness becomes harder).

Indeed, the assumption of allowing each BS to be a member of only one group may appear to be restrictive because by removing this assumption, i.e. allowing a base station to belong to more than one group, we increase efficiency: suppose that there is a base station which is not causing interference to any other base station, clearly this base station should belong to all groups. In the following, we investigate the truthfulness of the previous mechanisms when removing this restriction, by addressing the following question: given the allocation rule and the hypothesis that a player (BS) can belong to more than one group, is there a payment rule such that those mechanisms are still truthful? 
We shall add a star to the original mechanism to denote the new version. Note that the difference between Mechanism and Mechanism* (if it exists) resides only in the payment rule.

\subsection{Candidate mechanisms are not all adaptable}

In this subsection, we show that we can adapt all previous mechanisms except TAMES and TLSAA2.

Before that, let us introduce the following proposition.

Proposition 5. Given a monotone allocation rule, if a player belongs to all groups then he pays 0 .

Proof. Direct application of Myerson's lemma (there is no breaking point for this player because he is always a winning player).

We now analyse the extension of each one of the studied mechanisms to the case where assumption $\mathrm{A}$ is relaxed.

- TAMES:

Under TAMES, all players of the group with the highest groupbid are winners except the player with the lowest bid. With the assumption that a player can belong to more than one group, the allocation rule is non-monotone. Indeed, consider a player with valuation equal to 15 , belonging to two groups with bids respectively $\{\mathbf{1 5}, 20,25\}$ and $\{8,9, \mathbf{1 5}, 20\}$. Bidding truthfully leads to a utility equal to 0 because he is a loosing player. However, any bid lower than 12 leads to a higher utility because in that situation this player is a winning player. Since the allocation rule is not monotone anymore, we cannot find a truthful payment rule.

- TRUST $^{*}$

Under TRUST* all players of the group with the highest group bid are winners. Clearly, the allocation rule is monotone. Thus we can find a truthful payment rule. The breakpoint for player $i$ is given by the minimum bid that allows $i$ to win the auction: for each group $k$ which $i$ belongs to, we compute the minimum bid, if it exists, which allows him to win the auction.

- $\mathrm{VCG}^{*}$ :

We propose to adapt VCG in this context. The winning group is the group with the highest groupbid. We denote by $B_{\max }^{-i}$ the highest groupbid of groups to which $i$ does not belong. If the player belongs to the winning group $g_{\text {win }}$ with groupbid $B_{\text {win }}$ then we can distinguish two cases: if $i$ 's presence does not change the outcome i.e., $B_{\text {win }}^{-i} \geq B_{\max }^{-i}$ then he pays 0 otherwise he pays $B_{\max }^{-i}-B_{\text {win }}^{-i}$. To summarize:

$$
p_{i}^{V C G *}=\left[B_{\max }^{-i}-B_{w i n}^{-i}\right]^{+} .
$$

- PAM $^{*}$ :

We denote by $n_{i}$ the number of groups which $i$ belongs to. The initial version of PAM in [12] was ac- tually designed under this assumption. The payment rule is given by:

$$
\begin{aligned}
p_{i}= & \frac{n_{i} b_{i}+B_{g}^{-i}}{n_{i} b_{i}+B_{\mathrm{Tot}}^{-i}} R+\frac{B_{\mathrm{Tot}}^{-i}-B_{g}^{-i}}{n_{i}} \\
& \left(\ln \frac{n_{i} b_{i}+B_{\mathrm{Tot}}^{-i}}{n_{i} R+B_{\mathrm{Tot}}^{-i}}+\frac{n_{i} R+B_{\mathrm{Tot}}^{-i}}{n_{i} b_{i}+B_{\mathrm{Tot}}^{-i}}-1\right) .
\end{aligned}
$$

- TLSAA*

Under TLSAA* all players of the group with the highest groupbid are winners. Clearly, the allocation rule is monotone. Thus we can find a truthful payment rule. The breakpoint for player $i$ is given by the minimum bid that allows $i$ to win the auction: for each group $k$ which $i$ belongs to, we compute the minimum bid, if it exists, which allows him to win the auction.

- TLSAA2:

We cannot find a truthful payment rule since the allocation rule is non-monotone, which can be seen on the following example, with two groups with bids respectively $\{15,5,3,2.5\}$ and $\{7,5,4\}$. Clearly the player with the bid in bold (5) is a losing player (the first group wins the auction and only the first player is a winning player and he pays 12). However, if player $i$ proposed $b_{i}=2.5$ instead of 5 then he would be a winning player because in this situation all players of the first group would be winners and each one pays 2 .

Table 3 summarizes the different truthful mechanisms for LSA with and without Assumption A.

\begin{tabular}{|l|l|}
\hline Scenario & $\begin{array}{l}\text { Candidate truthful mecha- } \\
\text { nisms }\end{array}$ \\
\hline \hline $\begin{array}{l}\text { Each base station belongs to one } \\
\text { and only one group }\end{array}$ & $\begin{array}{l}\text { TAMES, TRUST, TLSAA, } \\
\text { TLSAA2, VCG, PAM }\end{array}$ \\
\hline $\begin{array}{l}\text { Each base station, can belong to } \\
\text { more than one group }\end{array}$ & $\begin{array}{l}\text { TRUST* }{ }^{*} \text { TLSAA*, VCG } \\
\text { PAM }^{*}\end{array}$ \\
\hline
\end{tabular}

TABLE 3: Candidate truthful mechanisms.

In the following we numerically evaluate the impact of Assumption A. we compare $\mathrm{Mec}$ and $\mathrm{Mec}^{*}$, without considering TAMES and TLSAA2 since as we have shown they can not be extended preserving truthfulness.

\subsection{Numerical evaluation}

In this section we study the impact of assumption $\mathrm{A}$ on each mechanism. Then for each mechanism we select the reserve price and the configuration which maximizes the utility of the regulator for a fixed value of $\beta_{1}, \beta_{2}$ and $\beta_{3}$. $\boldsymbol{\beta}$ vector and its components were defined in Section 3 . We have done the following simulations: we have fixed two possible group configurations from the interference graph of Fig. 3: In the first configuration $C_{1}$, we have two groups $g_{1}=\{1,2,4,6\}$ and $g_{2}=\{3,5\}$. For the second configuration $C_{2}$ we have three groups $g_{1}=\{1,2,4,6\}, g_{2}=\{1,2,5\}$ and $g_{3}=\{3,5,6\}$. The marginal valuations of base stations are drawn from the uniform distribution over the interval $[0,100]$. For each mechanism and for each reserve price $R$, we have computed the average (with respect to each metric) over 10000 draws. 


\subsubsection{Impact of assumption $A$ on the previous mechanisms}

Results are shown on Figs. 7 to 10. As we can see from these figures, for the all-or-nothing mechanisms, efficiency and fairness are higher when a player can belong to more than one group: the cardinal of the winning group will be higher in average with this assumption, yielding larger fairness and efficiency. Hence first conclusion is: if the regulator wants to adapt an all-or-nothing mechanism and his objectives are to maximize efficiency and fairness, he should choose $\mathrm{Mec}^{*}$ instead of Mec, i.e., construct groups by allowing base stations to belong to several groups.

When the allocation is not all-or-nothing (PAM and $\mathrm{PAM}^{*}$ ), it seems to be natural that efficiency and fairness have opposite trends. First, if we increase efficiency then we decrease fairness in average. Indeed, increasing efficiency means that, in average, players with the highest valuations obtain more allocation (and hence players with lower valuations obtain less), i.e., the allocation gap between players increases hence a smaller Jain index (fairness).

In terms of revenue, as we can see from those figures for some reserve prices the revenue generated by $\mathrm{Mec}$ is higher than the revenue generated by $\mathrm{Mec}^{*}$, and we have the opposite for other reserve prices. Thus we are not able to conclude whether $\mathrm{Mec}^{*}$ is better than $\mathrm{Mec}^{*}$ (or the opposite) in terms of revenue.
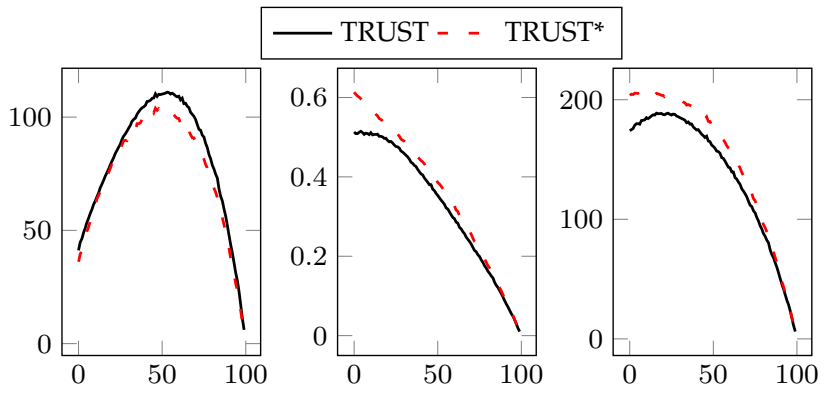

Fig. 7: Average revenue (left), fairness (center) and efficiency as a function of the reserve price.
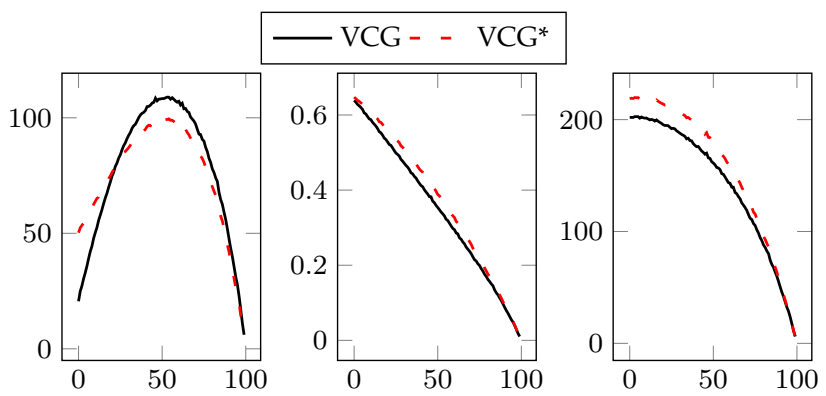

Fig. 8: Average revenue (left), fairness (center) and efficiency (right) as a function of the reserve price.

\subsubsection{Impact of assumption $A$ on the utility of the regulator}

Table 4 shows, for a fixed value of $\boldsymbol{\beta}$, how the regulator can maximize his utility by choosing a specific mechanism and by applying an appropriate configuration and reserve price. Also we provide the gain of the operator when assumption A is relaxed i.e., how much his utility is increased compared
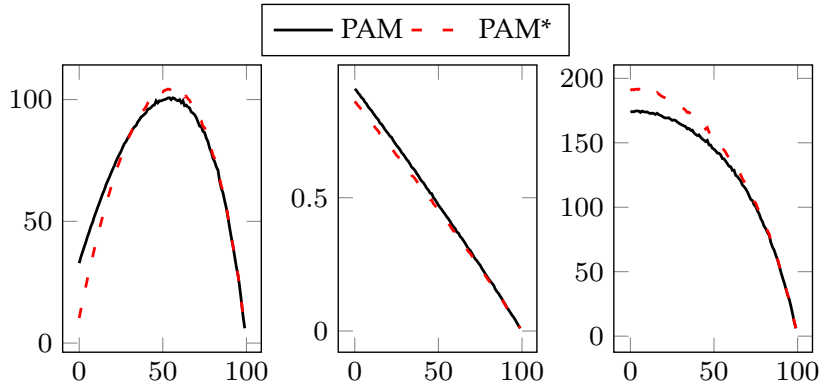

Fig. 9: Average revenue (left), fairness (center) and efficiency (right) as a function of the reserve price.

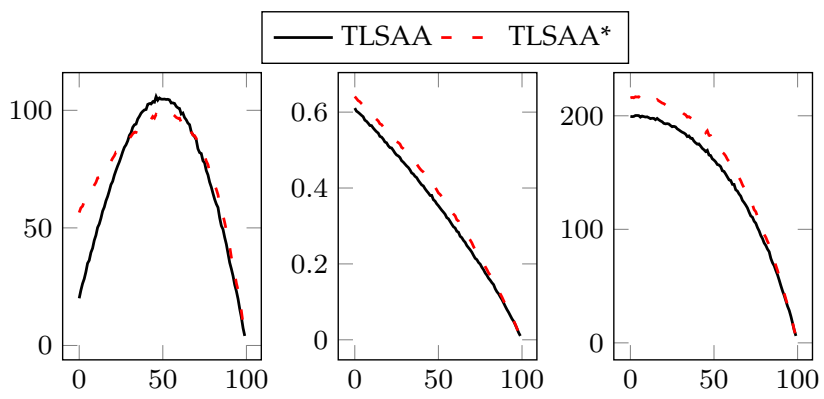

Fig. 10: Average revenue (left), fairness (center) and efficiency (right) as a function of the reserve price.

\begin{tabular}{llllll}
\hline$\left(\beta_{1}, \beta_{2}, \beta_{3}\right)$ & Mechanism & Configuration & $R$ & $U_{\text {Reg }}$ & Gain \\
\hline$\left(\frac{1}{3}, \frac{1}{3}, \frac{1}{3}\right)$ & VCG $^{*}$ & $C_{2}$ & 27 & 0.765 & $8 \%$ \\
\hline$(0.5,0.5,0)$ & PAM $^{*}$ & $C_{2}$ & 46 & 0.7384 & $4 \%$ \\
\hline$(0.5,0,0.5)$ & TRUST $^{*}$ & $C_{2}$ & 41 & 0.92 & $9.5 \%$ \\
\hline$(0,0.5,0.5)$ & PAM $^{*}$ & $C_{2}$ & 0 & 0.8638 & $3 \%$ \\
\hline$(1,0,0)$ & PAM $^{*}$ & $C_{2}$ & 56 & 1 & $2 \%$ \\
\hline$(0,1,0)$ & PAM $^{*}$ & $C_{1}$ & 0 & 0.94 & $\times$ \\
\hline$(0,0,1)$ & VCG $^{*}$ & $C_{2}$ & 0 & 1 & $23 \%$ \\
\hline$(0.2,0.2,0.6)$ & TLSAA & $C_{2}$ & 16 & 0.845 & $14 \%$ \\
\hline
\end{tabular}

TABLE 4: Optimal mechanisms, reserve prices and configuration for some specific values of $\beta_{1}, \beta_{2}$ and $\beta_{3}$.

to the scenario in which there is only $C_{1}$. In most cases the extension increases the utility of the regulator except when his objective is to maximize the fairness of the allocation only: as mentioned before, PAM is the most fair mechanism and it works better when each base station belongs to one and only one group.

\section{Conclusion}

In this paper, we have designed new truthful auction mechanisms aimed at allocating spectrum in the context of LSA. We have also studied the impact of an hypothesis found in all the literature, i.e. "each base station must belong to one and only one group" on truthfulness and we have extended previous studied mechanisms to the scenario in which this hypothesis is relaxed by finding the corresponding payment rule (if it exists) eliciting truthful bidding. The studied mechanisms have different properties so the regulator can choose one with respect to his preferences.

We have focused on sealed auctions i.e., all bidders simultaneously submit sealed bids. In future works, we will rather focus on ascendant open auctions; since they preserve 
the privacy of the winning bidder because the winner does not reveal his valuation and they give bidders the opportunity to adjust their valuations over the convergence phase. We will also study the case in which the regulator has more than one block to allocate, which complicates the auction analysis.

\section{REFERENCES}

[1] X. Ge, S. Tu, G. Mao, C.-X. Wang, and T. Han, "5g ultra-dense cellular networks," IEEE Wireless Communications, vol. 23, no. 1, pp. 72-79, 2016.

[2] M. Matinmikko, H. Okkonen, M. Malola, S. Yrjola, P. Ahokangas, and M. Mustonen, "Spectrum sharing using licensed shared access: the concept and its workflow for LTE-advanced networks," IEEE Wireless Communications, vol. 21, pp. 72-79, May 2014.

[3] A. Amraoui, B. Benmammar, and F. Bendimerad, "Accès Dynamique au Spectre dans le Contexte de la Radio Cognitive," in zème édition de la conférence nationale de l'informatique destinée aux étudiants de graduation et de post-graduation, (Alger, Algérie), Apr 2012.

[4] Z. Sadreddini, P. Masek, T. Cavdar, A. Ometov, J. Hosek, I. Gudkova, and S. Andreev, "Dynamic resource sharing in $5 \mathrm{~g}$ with lsa: Criteria-based management framework," Wireless Communications and Mobile Computing, vol. 2018, 2018.

[5] E. C. Committee, "ECC Report 205." Available at http://www. erodocdb.dk/Docs/doc98/official/pdf/ECCREP205.PDF", 2014.

[6] V. Frascolla, A. J. Morgado, A. Gomes, M. M. Butt, N. Marchetti, K. Voulgaris, and C. B. Papadias, "Dynamic licensed shared access-a new architecture and spectrum allocation techniques," in Proc. of IEEE VTC Fall, pp. 1-5, 2016.

[7] S. Yrjølå and H. Kokkinen, "Licensed shared access evolution enables early access to $5 \mathrm{~g}$ spectrum and novel use cases," EAI Endorsed Transactions on Wireless Spectrum, vol. 3, 122017.

[8] R. B. Myerson, "Optimal auction design," Mathematics of operations research, vol. 6, no. 1, pp. 58-73, 1981.

[9] V. Krishna, Auction Theory. Academic Press, 2009.

[10] R. Jain, D. M. Chiu, and W. Hawe, "A quantitative measure of fairness and discrimination for resource allocation in shared computer systems," CoRR, vol. cs.nI/9809099, 1998.

[11] H. Wang, E. Dutkiewicz, G. Fang, and M. Dominik Mueck, "Spectrum Sharing Based on Truthful Auction in Licensed Shared Access Systems," in Vehicular Technology Conference, Jul 2015.

[12] A. Chouayakh, A. Bechler, I. Amigo, L. Nuaymi, and P. Maillé, "PAM: A Fair and Truthful Mechanism for 5G Dynamic Spectrum Allocation," in Proc. of IEEE PIMRC, 2018.

[13] M. J. Osborne and A. Rubinstein, A Course in Game Theory. MIT Press, 1994.

[14] Z. Li, B. Li, and Y. Zhu, "Designing truthful spectrum auctions for multi-hop secondary networks," IEEE Transactions on Mobile Computing, vol. 14, no. 2, pp. 316-327, 2015.

[15] I. A. Kash, R. Murty, and D. C. Parkes, "Enabling spectrum sharing in secondary market auctions," IEEE Transactions on Mobile Computing, vol. 13, no. 3, pp. 556-568, 2014.

[16] R. B. Myerson, "Optimal auction design," Math. Oper. Res., vol. 6, pp. 58-73, Feb. 1981.

[17] P. Maillé and B. Tuffin, Telecommunication network Economics. Cambridge University Press, 2014.

[18] R. K. Jain, D.-M. W. Chiu, and W. R. Hawe, "A quantitative measure of fairness and discrimination," Eastern Research Laboratory, Digital Equipment Corporation, Hudson, MA, 1984.

[19] T. Roughgarden and M. Sundararajan, "Is efficiency expensive," in Third Workshop on Sponsored Search Auctions, 2007.

[20] E. Maskin, Auctions and Efficiency, pp. 1-24. Cambridge University Press, 2003. (text of the Seattle lecture).

[21] T. Roughgarden, Myerson's Lemma, p. 24-38. Cambridge University Press, 2016.

[22] A. Chouayakh, A. Bechler, I. Amigo, P. Maillé, and L. Nuaymi, "Auction mechanisms for Licensed Shared Access: reserve prices and revenue-fairness tradeoffs," in Proc. of IFIP WG PERFORMANCE, 2018.

[23] X. Zhou, S. Gandhi, S. Suri, and H. Zheng, "eBay in the sky: Strategy-proof wireless spectrum auctions," in Proc. of ACM MobiCom, pp. 2-13, 2008.
[24] W. Wang, B. Liang, and B. Li, "Designing truthful spectrum double auctions with local markets," IEEE Transactions on Mobile Computing, vol. 13, no. 1, pp. 75-88, 2014.

[25] Y. Chen, J. Zhang, K. Wu, and Q. Zhang, "Tames: A truthful auction mechanism for heterogeneous spectrum allocation," in IEEE INFOCOM, pp. 180-184, May 2013.

[26] X. Zhou and H. Zheng, "Trust: A general framework for truthful double spectrum access," in Proc. of IEEE INFOCOM, 2009.

[27] W. Vickrey, "Counterspeculation, auctions, and competitive sealed tenders," The Journal of finance, vol. 16, no. 1, pp. 8-37, 1961.

[28] E. H. Clarke, "Multipart pricing of public goods," Public choice, vol. 11, no. 1, pp. 17-33, 1971.

[29] T. Groves, "Incentives in teams," Econometrica: Journal of the Econometric Society, pp. 617-631, 1973.

[30] H. Fu, "Vcg auctions with reserve prices: Lazy or eager," EC, 2013 Proceedings ACM, 2013.

\section{APPENDIX A \\ PAM'S PAYMENT WHEN INTRODUCING A MINIMUM AMOUNT $\alpha$ OF SPECTRUM PER WINNER}

We propose to develop the analytical expression of the payment rule when introducing a minimum fraction $\alpha$. Roughgarden shows how to compute the payment when the allocation is a monotone function [21]: it is equal to the area bounded by the allocation curve and $y=\alpha_{i}$. As an example, without that minimum amount, the traditional payment rule of PAM is represented by the colored area in Fig. 11.

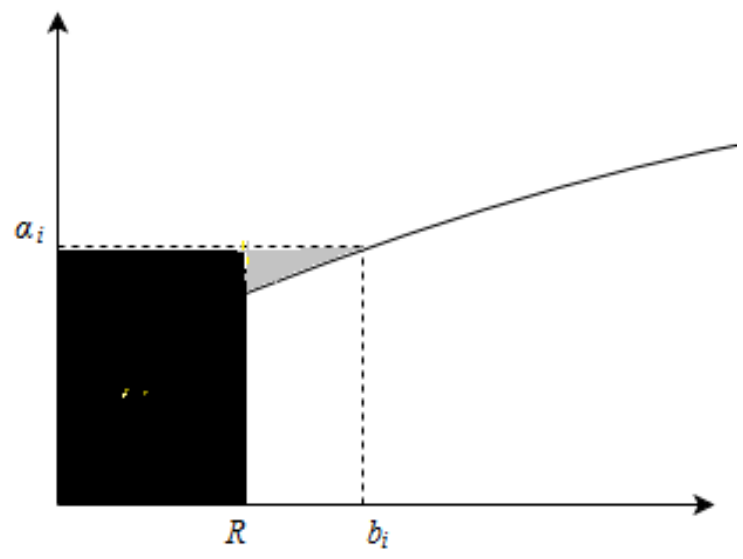

Fig. 11: Payment of PAM: the black surface is the first term of the payment $\left(\frac{b_{i}+B_{g}^{-i}}{b_{i}+B_{\text {Tot }}^{-i}} R\right)$, the rest of payment, denoted by $S_{i}\left(B_{\text {Tot }}^{-i}, R, B_{g}^{-i}, b_{i}\right)$, is represented by the grey surface and equals $S_{i}\left(B_{\mathrm{Tot}}^{-i}, R, B_{g}^{-i}, b_{i}\right)=\left(B_{\mathrm{Tot}}^{-i}-B_{g}^{-i}\right) \times$ $\left(\ln \left(\frac{b_{i}+B_{\text {Tot }}^{-i}}{R+B_{\mathrm{Tot}}^{-i}}\right)+\frac{R+B_{\mathrm{Tot}}^{-i}}{b_{i}+B_{\mathrm{Tot}}^{-i}}-1\right)$.

Let us fix a player $i$, we denote by $b_{i}^{*}$ the minimum bid that allows him to obtain $\alpha$, if $b_{i}^{*}$ is lower than $R$ then we set it to $R$ (and he gets more than $\alpha$ ). We sort group bid of other group in a non-decreasing order. Player $i$ can exclude some groups by increasing his bid. We suppose that player $i$ can exclude $m_{i}$ groups, i.e. there are $(M-1)-m_{i}$ groups excluded without the participation of player $i$. Without loss of generality, we suppose that player $i$ can exclude the first $m_{i}$ groups (which are sorted in a non- decreasing order). We denote by $\left\{c_{1}, \ldots, c_{m_{i}}\right\}$, the breaking points, i.e. if player 
$i$ proposes a bid higher that $c_{j}$ then group $j$ (and groups below) will be excluded. Also, $B_{\text {Tot }}^{-i}$ is rather $B_{\text {Tot }}^{-i}\left(b_{i}\right)$. We denote by $\left(B_{\text {Tot }}^{-i}\right)^{0}$ the sum of all group bid except the bid of player $i$, when player $i$ does not exclude any group (of the $m_{i}$ groups that he can exclude). We denote by $\left(B_{\text {Tot }}^{-i}\right)^{j}$ the sum of all bids after excluding group $j$ (and groups below), $\left(B_{\text {Tot }}^{-i}\right)^{j}=\left(B_{\text {Tot }}^{-i}\right)^{0}-\sum_{i=1}^{j} B_{i}$. Let us compute those breaking points: in order to exclude the first group the following conditions must hold:

1) $\frac{B_{1}}{\left(B_{\text {Tot }}^{-i}\right)^{0}+b_{i}}<\alpha$

2) $b_{i} \geq b_{i}^{*}$.

Thus, $c_{1}=\max \left\{b_{i}^{*}, \frac{B_{1}}{\alpha}-\left(B_{\text {Tot }}^{-i}\right)^{0}\right\}$. In general $c_{j}=$ $\max \left\{b_{i}^{*}, \frac{B_{j}}{\alpha}-\left(B_{\text {Tot }}^{-i}\right)^{j-1}\right\}$. We denote by $l$ the number of group excluded when $b_{i}=b_{i}^{*}$. The payment rule is given by:

$$
p_{i}\left(b_{i}\right)= \begin{cases}P_{i}^{\mathrm{PAM}}\left(b_{i},\left(B_{\mathrm{Tot}}^{-i}\right)^{l}, b_{i}^{*}\right), & \text { if } b_{i}^{*} \leq b_{i} \leq c_{l+1} \\ p_{i}\left(c_{k}\right)+S_{i}^{k}\left(b_{i}\right)+J_{i}^{k}\left(b_{i}\right) & \text { if } c_{k}<b_{i} \leq c_{k+1}, \\ & k \in \llbracket l+1 ; m-1 \rrbracket \\ p_{i}\left(c_{m_{i}}\right)+J_{i}^{m_{i}}, & \text { if } b_{i}>c_{m_{i}}\end{cases}
$$

Where:

- $\quad P_{i}^{\mathrm{PAM}}\left(b_{i},\left(B_{\text {Tot }}^{-i}\right)^{l}, b_{i}^{*}\right)$ is equal to Equation (5) by replacing $R$ by $b_{i}^{*}$ and $B_{\text {Tot }}^{-i}$ by $\left(B_{\text {Tot }}^{-i}\right)^{l}$

- $S_{i}^{k}\left(b_{i}\right)=S_{i}\left(\left(B_{\mathrm{Tot}}^{-i}\right)^{k}, c_{k}, B_{g}^{-i}, b_{i}\right)$

- $J_{i}^{k}\left(b_{i}\right)=c_{k}\left(\frac{b_{i}+B_{g}^{-i}}{b_{i}+\left(B_{\mathrm{Tot}}^{-i}\right)^{k}}-\frac{c_{k}+B_{g}^{-i}}{c_{k}+\left(B_{\mathrm{Tot}}^{-i}\right)^{k-1}}\right)$

- $J_{i}^{m_{i}}=c_{m_{i}}\left(1-\frac{c_{m_{i}}+B_{g}^{-i}}{c_{m_{i}}+\left(B_{\mathrm{Tot}}^{-i}\right)^{m_{i}-1}}\right)$

As an example if $c_{l+1}<b_{i} \leq c_{l+2}$ then $p_{i}\left(b_{i}\right)$ is equal to the sum of the following terms:

- $p_{i}\left(c_{l+1}\right)=$

$$
\begin{aligned}
& \frac{c_{l+1}+B_{g}^{-i}}{c_{l+1}+B_{\text {Tot }}^{-i}} b_{i}^{*}+\left(B_{\text {Tot }}^{-i}-B_{g}^{-i}\right) \\
& \left(\ln \left(\frac{c_{l+1}+B_{\text {Tot }}^{-i}}{b_{i}^{*}+B_{\text {Tot }}^{-i}}\right)+\frac{b_{i}^{*}+B_{\text {Tot }}^{-i}}{c_{l+1}+B_{\text {Tot }}^{-i}}-1\right),
\end{aligned}
$$

represented by the white area $\left(S_{1}\right)$ in Figure 12 .

- $J_{i}^{l+1}\left(b_{i}\right)=$

$$
c_{l+1}\left(\frac{b_{i}+B_{g}^{-i}}{b_{i}+\left(B_{\text {Tot }}^{-i}\right)^{l+1}}-\frac{c_{l+1}+B_{g}^{-i}}{c_{l+1}+\left(B_{\text {Tot }}^{-i}\right)^{l}}\right),
$$

represented by the black area in Figure 12 .

- $S_{i}^{l+1}\left(b_{i}\right)=$

$$
\begin{aligned}
& \left(\left(B_{\mathrm{Tot}}^{-i}\right)^{l+1}-B_{g}^{-i}\right) \times\left(\operatorname { l n } \left(\frac{b_{i}+\left(B_{\mathrm{Tot}}^{-i}\right)^{l+1}}{\left.c_{l+1}+B_{\mathrm{Tot}}^{-i}\right)^{l+1}}\right.\right. \\
& \left.+\frac{c_{l+1}+\left(B_{\mathrm{Tot}}^{-i}\right)^{l+1}}{b_{i}+\left(B_{\mathrm{Tot}}^{-i}\right)^{l+1}}-1\right),
\end{aligned}
$$

represented by the grey area in Figure 12 .

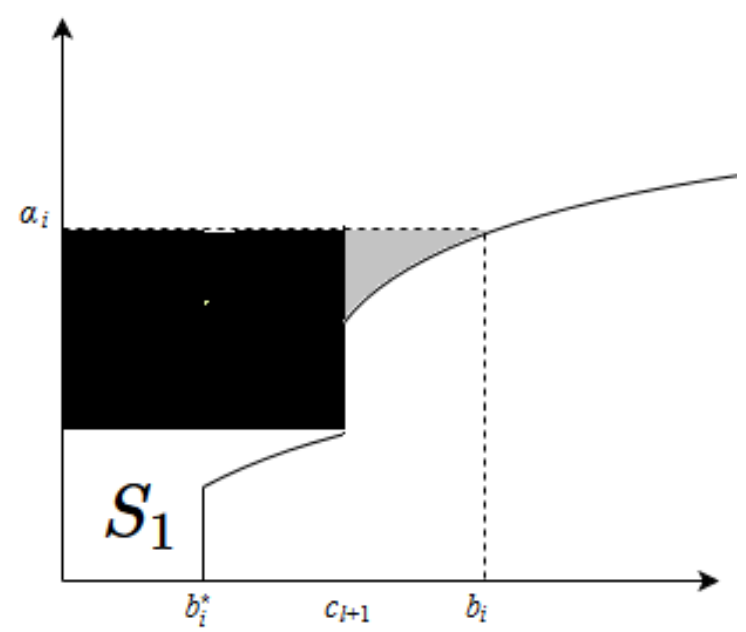

Fig. 12: Payment of player $i$ when $c_{l+1}<b_{i} \leq c_{l+2}$. 